\title{
Fabrication and photocatalytic properties of porous crystalline titanium dioxide film co-doped with Fe and Si by PEO
}

\author{
Miao Wang ${ }^{1, a,{ }^{*}}$, Weixin Ren ${ }^{1, b}$, Xiaoling Zhang ${ }^{1, b}$, Linzhong Zhu ${ }^{1, \mathrm{~d}}$, Jun Zhao ${ }^{1, \mathrm{e}}$, \\ Shujin Shi, ${ }^{1, \mathrm{f}}$, Qianqian $\mathrm{Liu}^{1, \mathrm{~g}}$, Shuang Guo ${ }^{1, \mathrm{~h}}$, Yunlong Wang ${ }^{1,1, *}$ \\ ${ }^{1}$ School of Materials Science and Engineering, Jiangsu University, Zhenjiang, 212013,China \\ a'Wangmiao@ujs.edu.cn(corresponding author Email), ${ }^{b}$ Renwx@ujs.edu.cn, ${ }^{\mathrm{c} Z h a n g x l @ u j s . e d u . c n, ~}$ \\ dZhulz@163.com, 'Zhaoj@ujs.edu.cn, Shisj@ujs.edu.cn, Liuqq@ujs.edu.cn, Guos@ujs.edu.cn, \\ Wyl306wm@ujs.edu.cn (Co-corresponding author Email)
}

Keywords: Porous titania film; Photocatalytic property; Plasma electrolytic oxidation; Carbon steel

\begin{abstract}
Porous photocatalytic titania film co-doped with Fe and Si was fabricated on carbon steel substrate by plasma electrolytic oxidation (PEO). The microstructure of the film was investigated and the photocatalytic activity of the film was tested by measuring the photodegradation rate of the organic methylene blue (MB). The results revealed that the obtained film was composed of anatase and rutile $\mathrm{TiO}_{2}$ crystalline phases. Fe and Si were co-doped in the film. The film was thick with the thickness of about $25 \mu \mathrm{m}$. It was double layered with a compact inner layer and loose out layer. The film surface was coarse and porous and was covered with micro pores. The photodegradation rate of MB reached $86 \%$ with 3 h irradiation of UV, showing a high photocatalytic activity. The method reports a rapid and simple route to fabrication porous crystalline $\mathrm{TiO}_{2}$ film with high photocatalytic activity.
\end{abstract}

\section{Introduction}

$\mathrm{TiO}_{2}$ film has received much and increasing attention due to its wide applications, among which the photocatalytic ability is one of the significant application. It can be used to degrade organic pollutants under the irradiation of ultraviolet lights [1-2]. This also gain increasing attentions during the situation that more concerns of human are concentrated on the environment and water pollution. Various methods have been developed to fabricated $\mathrm{TiO}_{2}$ films, such as sol-gel methods [3], chemical vapor deposition [4], Sputtering [5]. However, in most cases, a relatively high temperature are usually needed during preparation and the whole substrate are heated, or else amorphous $\mathrm{TiO}_{2}$ films will be often obtained when the temperature is high enough, which will usually decrease the photocatalytic activity。

The present paper reports the fabrication of photocatalytic crystalline $\mathrm{TiO}_{2}$ film in facile room temperature environment by plasma electrolytic oxidation (PEO) [6-8]. PEO is based on conversional anodic oxidization technology and is capable of preparing protective and functional films on metals. It is an eco-friendly technology which is widely used. In order to improve the performance of the $\mathrm{TiO}_{2}$ film, Fe and Si elements were co-doped into the $\mathrm{TiO}_{2}$ film by using $\mathrm{Fe}$ as substrate which was also used as Fe source and $\mathrm{Na}_{2} \mathrm{SiO}_{3}$ in electrolyte was used as Si source. The microstructure and the photolytic activity of the film were evaluated.

\section{Experimental details}

The conventional bi-electrode PEO system was adopted for preparation of the film. Q235 carbon steels with the dimension of $10 \mathrm{~mm} \times 20 \mathrm{~mm} \times 1.8 \mathrm{~mm}$ served as anode and a rectangle stainless steel sheet with the dimension of $20 \mathrm{~mm} \times 200 \mathrm{~mm} \times 1.8 \mathrm{~mm}$ was used as the counter electrode. A stainless steel electro-bath was used as the container of the electrolyte which was cooled by running water travelling around. A home-made electrical single-pulse power source was connected to the two electrodes. During PEO process, the parameters were: the current density was kept constant at 12A/dm2; the pulse frequency was kept $1000 \mathrm{~Hz}$; the treatment time was $10 \mathrm{~min}$. The electrolyte 
was silicate solution made up of some $\mathrm{Na}_{2} \mathrm{SiO}_{3}$, some $\mathrm{NaH}_{2} \mathrm{PO}_{2}$ and some $\mathrm{TiO}_{2}$ power. The carbon steels substrate was polished and thoroughly washed for

The phase composition of the film was detected by X-ray Diffractometer (XRD, D8 ADVANCE, Germany).The surface and cross section morphologies of the film were observed by scanning electron microscopy (SEM, S-3400N, Hitachi, Japan). The diameter distributions of the surface pores was calculated by the software Nana Measurer. The elements composition of the film was detected by the Energy Disperse Spectroscopy (EDS, accessory of SEM, USA).

The photocatalytic activity of the film was tested by measuring the photodegradation rate of methylene blue. A beaker of $100 \mathrm{ml}$ methylene blue (MB) solution is prepared and the initial concentration is $5 \mathrm{mg} / \mathrm{L}$. $\mathrm{TiO}_{2}$ film sample was immersed into the beaker and was illuminated perpendicularly by a germicidal lamp $(40 \mathrm{~W}, 254 \mathrm{~nm}$ wavelength $)$.The whole test lasted for $3 \mathrm{~h}$, and the concentration change of $\mathrm{MB}$ are measured every 30 minutes. The concentration of $\mathrm{MB}$ was determined using a UV spectrophotometer (UV-2450, Shimadz, Japan), by measuring the absorbance at $662 \mathrm{~nm}$, which show a linear and positive relationship with the concentration. The photodegradation rate of $\mathrm{MB}$ is measured by removal ratio

$$
\eta=\frac{C_{0}-C_{t}}{C_{0}} * 100 \%
$$

where $\eta$ is the degradation rate, $C_{0}$ is the initial absorbency and $C_{t}$ is absorbency of $\mathrm{MB}$ at a certain time.

\section{Results and discussion}

Fig.1 shows the surface and cross section morphologies of the films tested by SEM. Fig. 1a reveals that the film surface is porous and coarse. There are many micro pores on the film surface and the smallest pore is about $1 \mathrm{um}$. The pores are formed during the discharge reaction in PEO. They are the discharge channels and the porous surface is the typical structure of PEO films [6-7]. Fig 1a also indicates that as a whole the surface of the film is homogeneous. Few large embossments and concaves are presented on the spectrum. Fig.1b is the cross section morphology of the film. It reveals that the interface between the film and the steel substrate is clear but there is no evident gap or micro cracks between the film and the steel substrate. This reveals that the film is tightly growth on the substrate. It also displays that the film is very thick with the thickness of about $25 \mu \mathrm{m}$. In addition, a clear boundary can be found on the film cross section which separates the film into two layers, namely a relatively compact inner layer and a loose outer layer. This is also the typical characters of PEO film in the cross section.
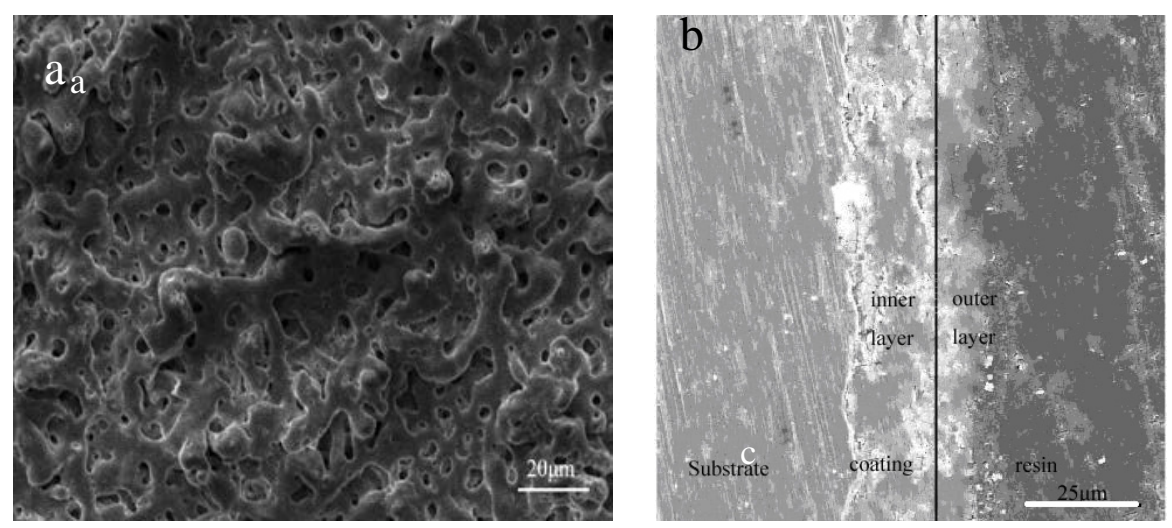

Fig 1. Surface morphology (a ) and cross section morphology (b) of the film

Fig. 2 shows the phase and elements composition of the obtained film. Fig. 2a reveals that the film is crystalline $\mathrm{TiO}_{2}$ containing both anatase and rutile. No peaks of element iron are found in the spectra. As pointed out above, the film is thick and is compact in the inner layer. The X-Ray can not penetrate through the film into the substrate, so no peaks of element iron are found .Fig. $2 b$ denotes that the apart from $\mathrm{Ti}$ and $\mathrm{O}$ elements which exist in $\mathrm{TiO}_{2}$, other elements like $\mathrm{Fe}$ and $\mathrm{Si}$ are 
also found in the coating [8]. The loose outer layer is porous and coarse which can be seen from the surface morphology.

Furthermore, a little $\mathrm{p}$ is also pound in coating, which is evidently from the electrolyte ingredient of $\mathrm{NaH}_{2} \mathrm{PO}_{2}$. According XRD spectra, the elements might be in the amorphous form of $\mathrm{Fe}_{3} \mathrm{O}_{4}$ and $\mathrm{SiO}_{2}$. There, the film is crystalline $\mathrm{TiO}_{2}$ containing both anatase and rutile and is co-doped by Fe and Si.
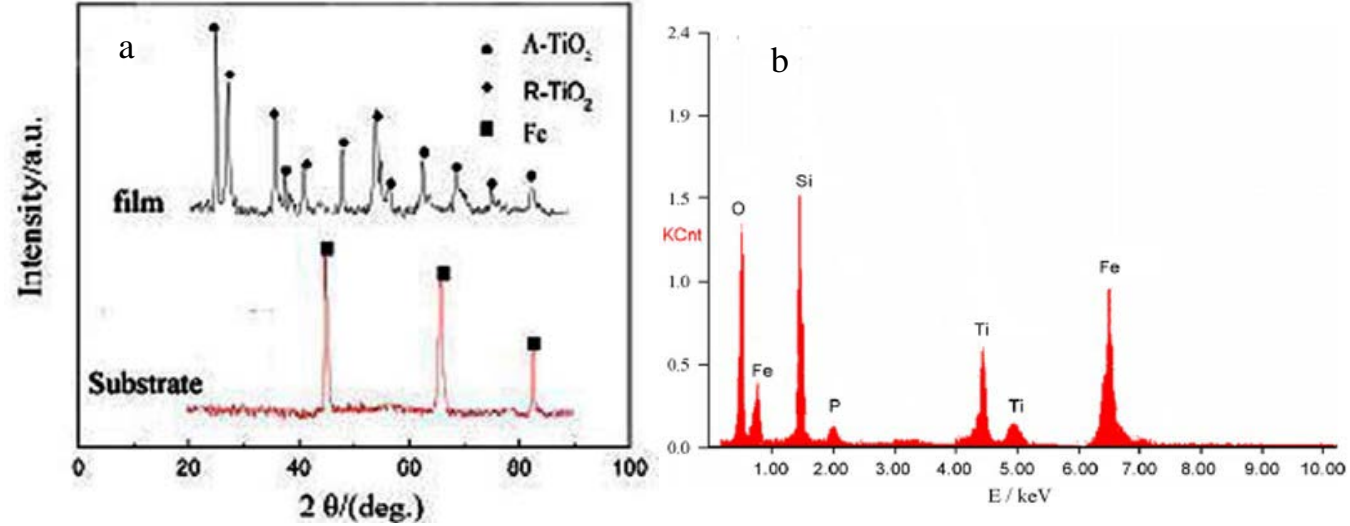

Fig.2. XRD (a) and EDS(b) spectra of the film

The removal ratio of $\mathrm{MB}$ of the composite $\mathrm{TiO}_{2}$ film during irradiation of UV light is described in Fig. 3. It can be seen that $\mathrm{TiO}_{2}$ films show effective photodegradation of MB. A high removal ratio of nearly $70 \%$ can be reached during about $1 \mathrm{~h}$ and a half irradiation. For $3 \mathrm{~h}$ irradiation, a total photodegradation ratio of $86 \%$ can be obtained. Therefore, the films obtained by PEO on steels show high photocatalytic activity. The photocatalytic activity of the film is attributed to the composition and structure of the film. First, the film is composed of anatase and rutile $\mathrm{TiO}_{2}$. It is reported that anatase $\mathrm{TiO}_{2}$ is active phase in photocatalytic activity and anatase accompanied with some rutile will further increase the photocatalytic activity [9]. Second, some Si and Fe in the film might also contribute to photocatalytic activity of the film. As it is reported, Fe or Si doped $\mathrm{TiO}_{2}$, exhibits higher photocatalytic activity [10-11]. Third, the microstructure of the film, namely porous surface, is also significant factor for the high catalytic activity. Porous surface offers large high surface area for the composite $\mathrm{TiO}_{2}$ photocatalyst, which forms a large number of active site for photodegradation of MB

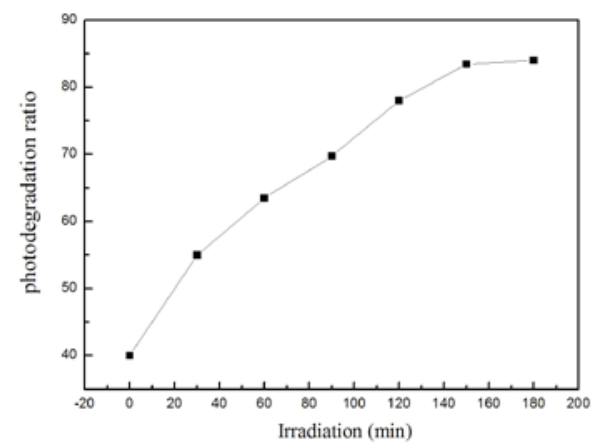

Fig.3. Photodegradation ratio of MB of the film

\section{Summary}

Porous photocatalytic titania film co-doped with $\mathrm{Fe}$ and $\mathrm{Si}$ was fabricated on carbon steel substrate by plasma electrolytic oxidation (PEO). The film was composed of anatase and rutile $\mathrm{TiO}_{2}$ crystalline phases. Fe and Si were co-doped in the film. The film was thick with the thickness of about $25 \mu \mathrm{m}$. It was double layered with a compact inner layer and loose out layer. The film surface was coarse and porous, and was covered with micro pores. The photodegradation rate of MB reached $86 \%$ with 3 h irradiation of UV, showing a high photocatalytic activity. 


\section{Acknowledgments}

This work was supported by the Natural Science Foundation of Jiangsu Province (BK20130509), the Natural science fund for colleges and universities in Jiangsu Province (12KJB430005), the Priority Academic Program Development of Jiangsu Higher Education Institutions. The corresponding author is YunlongWang, Tel/fax: 86-511-88790191, E-mail:wyl306wm@ujs.edu.cn. Wangmiao is a co-corresponding author, whose E-mail is wwangmiao@ujs.edu.cn

\section{References}

[1] Y.Z. Wang, H. Yang, X.X. Xue, Synergistic antibacterial activity of $\mathrm{TiO}_{2}$ co-doped with zinc and yttrium, Vacuum. 107 (2014) 28-32.

[2] W.X. Liu, P. Jiang, W.N. Shao, J. Zhang, W.B. Co, A novel approach for the synthesis of visible-light-active nanocrystalline $\mathrm{N}$-doped $\mathrm{TiO}_{2}$ photocatalytic hydrosol, Solid State Sci. 33 (2014) 45-48.

[3] X.Z. Yua, Z.G. Shen, Photocatalytic $\mathrm{TiO}_{2}$ films deposited on cenosphere particles by pulse magnetron sputtering method, Vacuum. 85 (2011) 1026-1031.

[4] K. Nakata, A. Fujishima, $\mathrm{TiO}_{2}$ photocatalysis: Design and applications, J. Photoch. Photobio. C, 13 (2012) 169-189.

[5] C. He, X.Z. Li , N. Graham, Y. Wang, Preparation of $\mathrm{TiO}_{2} / \mathrm{ITO}$ and $\mathrm{TiO}_{2} / \mathrm{Ti}$ photoelectrodes by magnetron sputtering for photocatalytic application, Appl. Catal., A. 305 (2006) 54-63.

[6] G.X. Shen, Y.C. Chen, C.J. Lin, Corrosion protection of $316 \mathrm{~L}$ stainless steel by a $\mathrm{TiO}_{2}$ nanoparticle coating prepared by sol-gel method, Thin Solid Films. 489 (2005) 130-136.

[7] Q.M. Wang , Se-Hun Kwon, Kwun Nam Hui, Doo-In Kim, Kwan San Hui, Kwang Ho Kim, Synthesis and properties of crystalline $\mathrm{TiO}_{2}$ films deposited by a HIPIMS+ technique, Vacuum. 89 (2013) 90-95.

[8] A.L. Yerokhin, X. Nie, A. Leyland, A. Matthews , S.J. Dowey, Plasma electrolysis for surface engineering, Surf. Coat. Technol. 122 (1999) 73-93.

[9] M.Wang, M; Y.L. Wang, B.Wu, Z.H. Jiang, Facile fix of porous composite titania photocatalytic film by PEO, Surf. Eng. DOI: http://dx.doi.org/10.1179/1743294414Y.0000000345

[11] D.M. Tobaldi, A. Tucci , G. Camera-Roda, G. Baldi, L. Esposito, Photocatalytic activity for exposed building materials, J. Eur. Ceram. Soc. 28 (2008) 2645-2652.

[12] H.J. Lin, T.S. Yang, C.S. Hsi, M.C. Wang, K.C. Lee, Optical and photocatalytic properties of $\mathrm{Fe}^{3+}$-doped $\mathrm{TiO}_{2}$ thin films prepared by a sol-gel spin coating, Ceram. Int. 40 (2014) 10633-10640. 